\title{
МОНИТОРИНГ ЭФФЕКТИВНОСТИ ПРОГРАММЫ ПРОФИЛАКТИКИ ЗАБОЛЕВАНИЙ, СВЯЗАННЫХ С ДЕФИЦИТОМ ЙОДА, В БРЯНСКОЙ ОБЛАСТИ
}

\author{
'Трошина Е.А., 'Маколина Н.П., 'Сенюшкина Е.С., 'Пронина Л.О., 'Никанкина Л.В., 'Малышева Н.М., \\ ${ }^{2}$ Оглоблина Г.Я., ${ }^{2}$ Фетисова А.В.
}

'ФГБУ «Национальный медицинский исследовательский чентр эндокринологии» Минздрава России, Москва, Россия ${ }^{2}$ Департамент здравоохранения Брянской области, Брянск, Россия

Брянская область относится к регионам Российской Федерации, в наибольшей степени пострадавшим в результате аварии на Чернобыльской атомной электростанции в 1986 г. В условиях хронического некомпенсированного дефицита йода в питании в первые месяцы после аварии происходил активный захват радиоактивного йода тканью щитовидной железы (ЩЖ), что неизбежно реализовалось в росте заболеваний щЖ у населения в последующем.

ЦЕЛЬ: оценка йодной обеспеченности населения Брянской области.

МАТЕРИАЛЫ И МЕТОДЫ: исследование проводилось в 2021 г. в общеобразовательных школах трех районов Брянской области (гг. Брянск, Новозыбков и Клинцы). В исследование были включены 337 школьников допубертатного возраста (8-10 лет), всем детям выполнено: измерение роста и веса, осмотр врачаэндокринолога с пальпацией ЩЖ; УЗИ ЩЖ с использованием портативного аппарата LOGIQе (China) с мультичастотным линейным датчиком 10-15 МГц; определение концентрации йода в разовых порциях мочи. Соответствие объема ЩЖ нормативным показателям, разработанным Zimmermann M. и соавт., оценивалось с учетом площади поверхности тела и пола детей. Определение концентрации йода в разовых образцах мочи $(\mathrm{n}=337)$ проводилось с помощью церий-арсенитного метода (на базе лаборатории ФГБУ «НМИЦ эндокринологии» Минздрава России). Качественное исследование на наличие йодата калия в образцах пищевой поваренной соли $(\mathrm{n}=344)$, полученной из домохозяйств и школьных столовых, осуществлялось на месте экспресс-методом. От всех родителей/опекунов детей получены информированные согласия на проведение обследования и обработку персональных данных. Разрешение локального этического комитета ФГБУ «НМИЦ эндокринологии» Минздрава России получено 25 марта 2020 г., протокол №5.

РЕзУЛЬТАТЫ: по результатам обследования 337 детей допубертатного возраста медианная концентрация йода в моче (мКЙМ) составляет 98,3 мкг/л (диапазон от 91,5 до 111,5 мкг/л, доля проб мочи со сниженной концентрацией йода составила 50,1\%). По данным УЗИ щЖ у 17\% обследованных детей был выявлен диффузный зоб, частота которого варьировала от 9,4 до $29 \%$ по областям исследования. Доля йодированной соли, употребляемой в семьях школьников районов исследования, составила 17,8\% (диапазон значений от 15,6 до 19\%). Вся соль, используемая для приготовления пищи в школьных столовых районов исследования, была йодированной, что подтверждает соблюдение требований СанПиН 2.4.5.2409-08.

ВЫВОды: результаты эпидемиологического исследования свидетельствуют о недостаточной обеспеченности йодом населения Брянской области: показатель мКЙМ составляет 98,3 мкг/л, поскольку доля домохозяйств, использующих йодированную соль, крайне низкая (17,8\%), что не соответствует рекомендациям ВО3 для регионов с природным дефицитом йода (от $90 \%$ и более).

В целом для Брянской области характерна легкая степень тяжести йододефицитных заболеваний (Йд3) с частотой распространения зоба у детей по данным УзИ в среднем 17\% (варьировала от 9,4 до 29\%).

Анализ полученных в ходе обследования данных выявил характерные для йододефицитных регионов закономерности: с нарастанием степени йодной недостаточности увеличивается частота зоба у детей младшего школьного возраста (наиболее неблагоприятная эпидемиологическая ситуация зафиксирована в Клинцовском районе, где доля зоба у детей составила 29\% при мКЙМ 91,5 мкг/л и низкой доле йодированной соли - 18,8\%). Проявления зобной эндемии более выражены у детей, проживающих в менее крупных населенных пунктах, чем у городских жителей (зоб по УзИ выявлен у 29 и 9,4\% детей соответственно), в этих же населенных пунктах уровень использования йодированной соли в пищу был ниже.

Несмотря на проведение в Брянской области различных профилактических мероприятий и реализацию целевых региональных программ, следует констатировать, что проблема ЙДЗ остается нерешенной в условиях отсутствия в регионе массовой профилактики при помощи йодированной соли.

КЛЮЧЕВЫЕ СЛОВА: йодный дефицит; зоб; йододефичитные заболевания; йодированная соль. 